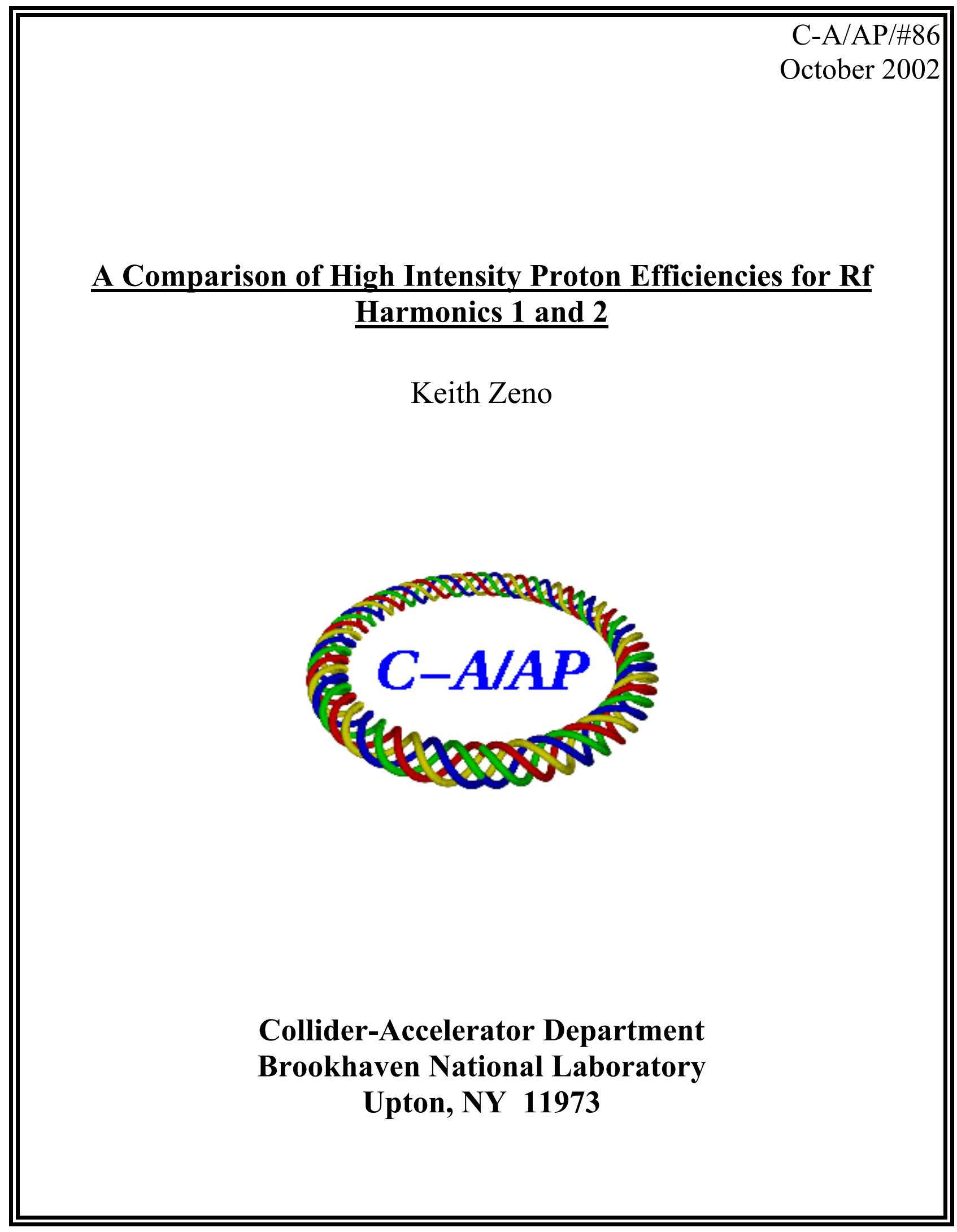




\section{A Comparison of High Intensity Proton Efficiencies for Rf Harmonics 1 and 2.}

Keith Zeno

August 27, 2002 


\section{Introduction}

There have been many changes to the Booster setup over the past seven years, but the most fundamental physical change is probably the change from an Rf harmonic of 2 to 1 . By looking at the intensity data over this period it is possible to compare the running efficiencies in each case. The harmonic change occurred at the beginning of the 1998 run. Another fundamental, though administrative, change that occurred after the 1998 run was the advent of ALARA alarms that limit the amount of beam loss that is allowed to occur during different parts of the cycle. These ALARA alarm limits also depend on the AGS repetition period since it is the amount of beam energy lost per unit time that is used as the criterion for these alarms. The $1998 \mathrm{~h}=1$ run did not have an ALARA constraint, but subsequent $\mathrm{h}=1$ runs did have that constraint.

The highest Booster proton intensity was obtained in the $\mathrm{h}=2$ mode (about $23 \mathrm{Tp}$ ). At best, the $h=1$ mode only yielded about $20 \mathrm{Tp}$ per cycle. Is it because the $h=2$ setup can produce higher intensity than the $h=1$ setup that the $h=2$ intensity was higher, or is it because other factors are responsible? This note seeks to shed some light on this question.

In order to compare the two setups $(\mathrm{h}=1 \mathrm{vs.} \mathrm{h}=2)$ intensity scaler data was taken from the setup books over the past seven years. In general, this data was taken when the Booster was in an optimized state. What 'optimized' means is not so clearly defined, but would typically mean that the Booster configuration was 'optimized' so that it yields the highest intensity late in the AGS given physical and other constraints.

\section{$\underline{\text { Analysis }}$}

Figure 1 shows the Booster input scaler value, which is the intensity coming from Linac to the Booster, versus the Booster early intensity scaler value. The early intensity scaler samples the Booster's circulating beam transformer early in the acceleration cycle. The injection efficiency (early/input) for the two cases is roughly similar until Booster input reaches about $30 \mathrm{Tp}$ at which point there are no more points for the $\mathrm{h}=1$ case. The Booster input reaches about $40 \mathrm{Tp}$ for the $\mathrm{h}=2$ case. Why are there no points for inputs higher than $30 \mathrm{Tp}$ for $\mathrm{h}=1$ ? The Linac intensity is not a limiting factor since Booster Input could be increased further without changing any longitudinal parameters (i.e.- pulse width, bunch width) by changing the percentage of 'sieve' used through the chopper program. Should one then infer that the AGS intensity and/or Booster early intensity do not increase if the input is increased further? If that were the case, one would naively expect the early $h=1$ intensity to at least show some sign of flattening out at the higher input values in the plot. Yet, the $h=1$ data is quite linear even at the highest inputs shown. The $h=2$ data does show some sign that it is gradually flattening out. Perhaps the flattening out is occurring at some latter stage in the cycle (i.e. - Booster acceleration, Booster to AGS transfer...).

The green line represents the injection ALARA limit for the last SEB run $(\mathrm{h}=1$ and rep. period $=5.4 \mathrm{sec}$ ). This was the $\mathrm{h}=1$ run with the least constraining ALARA limits.

Administratively, the Booster is not allowed to regularly run to the right of this line. It seems that the injection ALARA limit is not sufficient to account for limiting Booster input since there is some room on the red line that would allow running at higher input intensities if the behavior remained linear. 


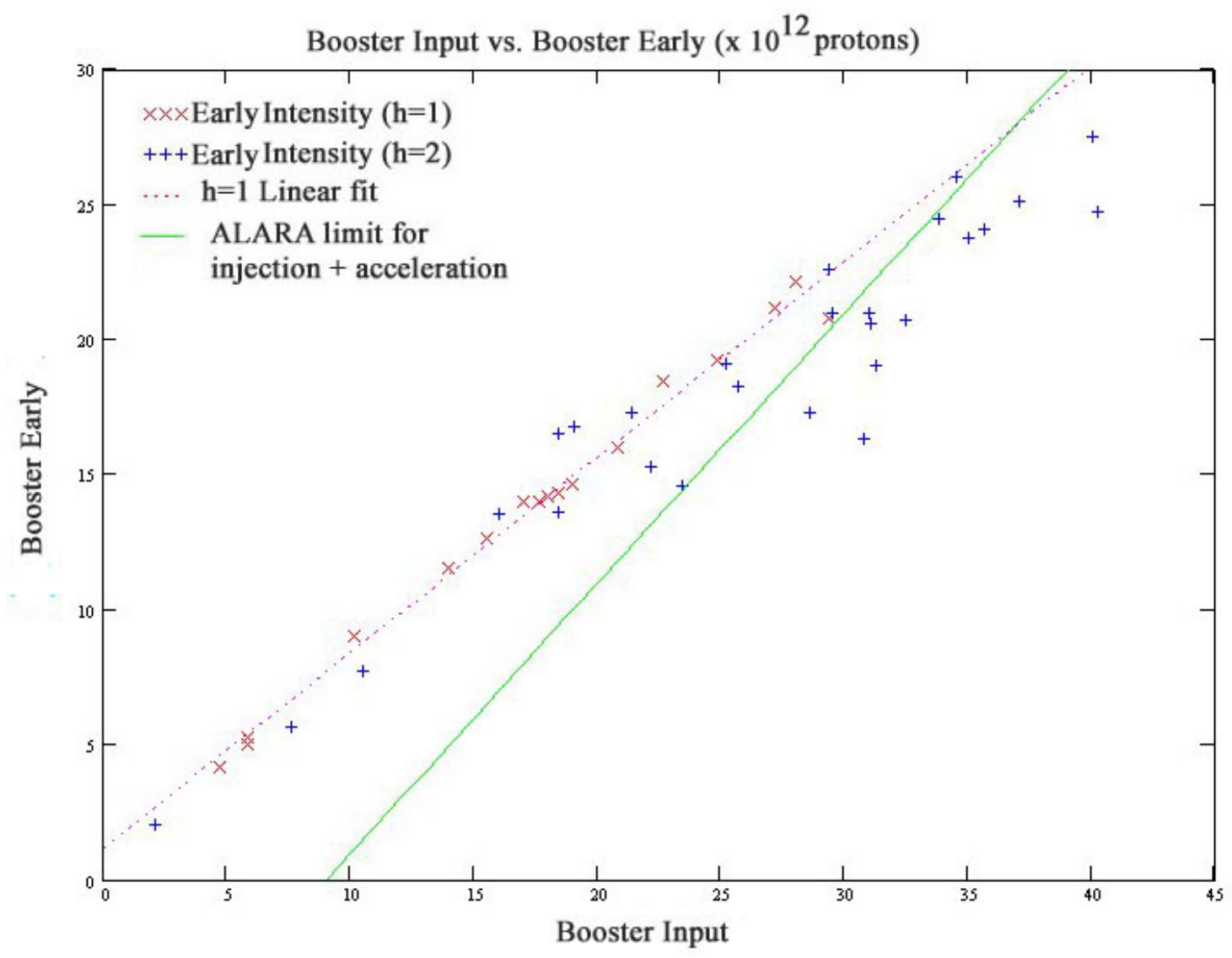

Figure 1: Booster Input vs. early intensity in the Booster for $\mathrm{h}=1$ (red) and $\mathrm{h}=2$ (blue) (per cycle). The $\mathrm{h}=1$ points are taken from the FY '98, '01, and '02 runs. The $\mathrm{h}=2$ points are taken from the FY '95, '96, and '97 runs. The red dotted line is a linear fits to the $\mathrm{h}=1$ data. The green line is the injection ALARA limit.

Figure 2 shows the acceleration losses for the two cases as well as the acceleration ALARA alarm limit for the 2002 run. The acceleration loss is the difference between the early and late intensity scaler readings. The late intensity scaler samples the Booster's circulating beam transformer late in the acceleration cycle. The acceleration losses for $\mathrm{h}=1$ are less than those for $h=2$. The acceleration loss for $h=1$ appears near the limit for an input of $30 \mathrm{Tp}$.

Figure 3 shows the input scaler versus the late intensity scaler value. Owing to the fact that acceleration losses are generally less with $h=1$ and injection losses may also be slightly less, the efficiency (late/input) is somewhat better for $h=1$ than for $h=2$, particularly at higher inputs. Although inconclusive, the combined injection and acceleration ALARA alarm limit is also shown. The $\mathrm{h}=1$ data is broken down further into the run from which it was taken. 


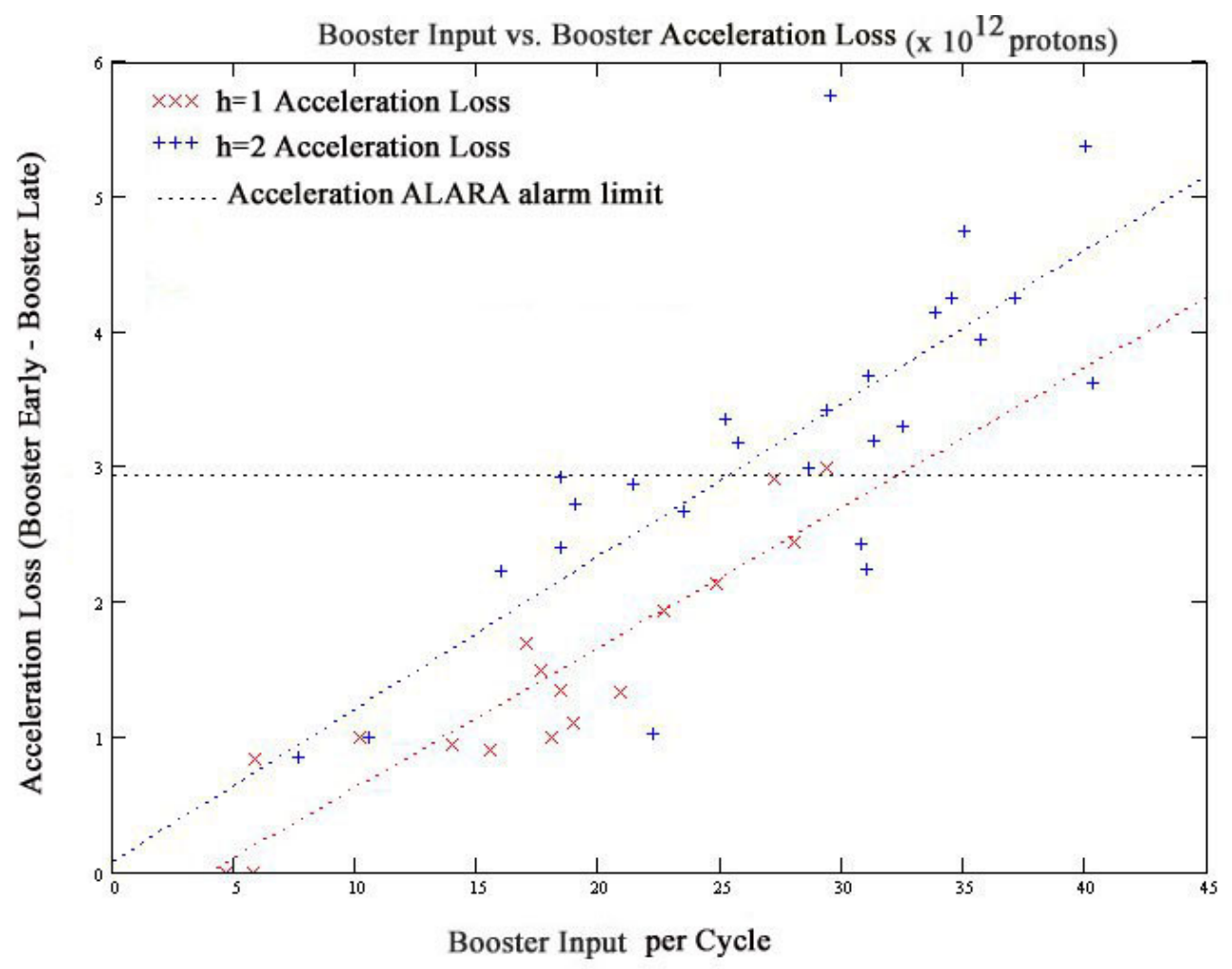

Figure 2: Booster Input vs. Booster acceleration losses for $h=1$ (red) and $h=2$ (blue). The $h=1$ points are taken from the FY ' 98 , ' 01 , and ' 02 runs. The $\mathrm{h}=2$ points are taken from the FY '95, '96, and '97 runs. The red and blue dotted lines are linear fits to this data. The dotted line is the acceleration ALARA limit for the last SEB run (FY' 02, 5.4 sec repetition period).

Late Intensity Vs. Booster Input (per cycle)

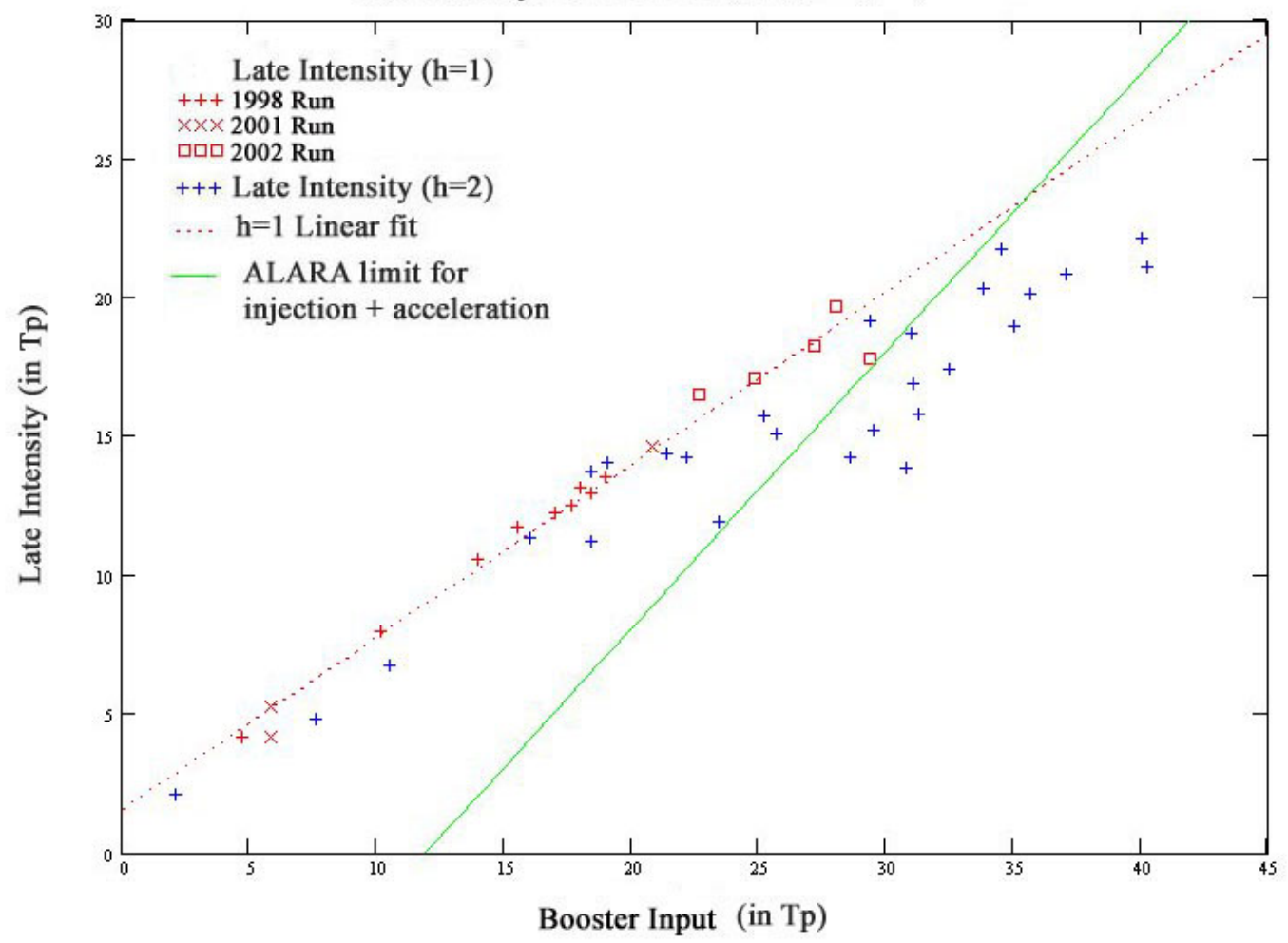

Figure 3: Booster Input vs. late Booster intensity for $\mathrm{h}=1$ (red) and $\mathrm{h}=2$ (blue). The $\mathrm{h}=1$ points are taken from the FY '98, '01, and '02 runs. The $\mathrm{h}=2$ points are taken from the FY '95, '96, and '97 runs. The red dotted line is a linear fit to the $\mathrm{h}=1$ data. The green line is the combined injection and acceleration ALARA limit for the 2002 run. 
Although much of the above data is quite linear, in general the $y$-intercepts of the linear fits do not pass through zero. Consequently, it would be a mistake to conclude that there is no intensity dependence, or flattening out of the data. For example, a linear fit that yields a small slope and a large y-intercept would indicate a large intensity dependence in that the efficiency, say (BoosterLate)/(BoosterInput), would get lower as the input is increased.

Focussing on the late intensity in particular (figure 3), it is somewhat puzzling that the data is so linear and yet the y-intercept is 1-2 Tp. An offset in the Booster late or input scaler could account for this, but an offset of 1-2 Tp x 6 cycles=6-12 Tp is unlikely. Particularly since this offset would have to be the same over several different runs. It is more likely that the dependence of Booster late on Booster Input is linear if the 'efficiency' is higher at lower intensity and does not decrease rapidly enough at higher inputs. For example, the $h=2$ data is does not look linear because the late intensity is flattening out faster, and there is a larger input range, than for the $\mathrm{h}=1$ data. There needs to be a distinction made between the Booster late vs. Booster input dependence, and the efficiency (i.e.- BoosterLate/Booster Input) dependence on Booster Input. It is the intensity dependence of the efficiency that we should be interested in. Additionally, the fact that there are not many data points at low intensity adds to the uncertainty in these matters.

Fortunately, the linearity (not the lack of intensity dependence) of the data can be used to show the intensity dependence of the efficiency (Booster Late/Booster Input). This efficiency is plotted in figure 4 . The red line in figure 4 is the transformation of the linear fit in figure 3 into a fit for the efficiency. That is,

\section{Efficiency $=\{m($ BoosterInput $)+b\} /$ BoosterInput}

where $\mathrm{m}$ is the slope of the linear fit, and $\mathrm{b}$ is the $\mathrm{y}$-intercept. Below an input of about $5 \mathrm{Tp}$ the fit gives efficiencies greater than 1 and is clearly not correct, but overall, the efficiency's intensity dependence is now apparent.

From figure 4 , the efficiency of $\mathrm{h}=2$ is generally somewhat less than it is for $\mathrm{h}=1$. Although there is intensity dependence in the $\mathrm{h}=1$ data, it does not appear sufficient to account for limiting the maximum $\mathrm{h}=1$ late intensity to $19-20 \mathrm{Tp}$ (or Booster Input to $30 \mathrm{Tp}$ ). A look at the Booster to AGS transfer is therefore in order.

\section{Booster to AGS Transfer Efficiency as a Function of Booster Late}

Firstly, let us look at the ALARA situation at the transfer. The transfer ALARA alarm limit was 38.4 Tp for the last SEB run. The highest $\mathrm{h}=1$ (or 2) AGS CBM number obtained during the run was about $79 \mathrm{Tp}$ (The AGS CBM measures the AGS intensity just after the last transfer has occurred). The highest late intensity during that run in the Booster was 118 Tp. The difference, (Booster late - AGS CBM), is 39 Tp, which is the ALARA limit. If, at this late intensity, the transfer efficiency (AGS CBM/BoosterLate) cannot be improved, then ALARA limits will constrain how much beam can be injected into the Booster and accelerated. This seems like the simplest explanation for why the Booster intensity for $h=1$ has not gone as high as for $h=2$. However, there are complications that arise when the situation is looked at more closely. 
Booster Input Vs. (Booster Late)/(Booster Input)

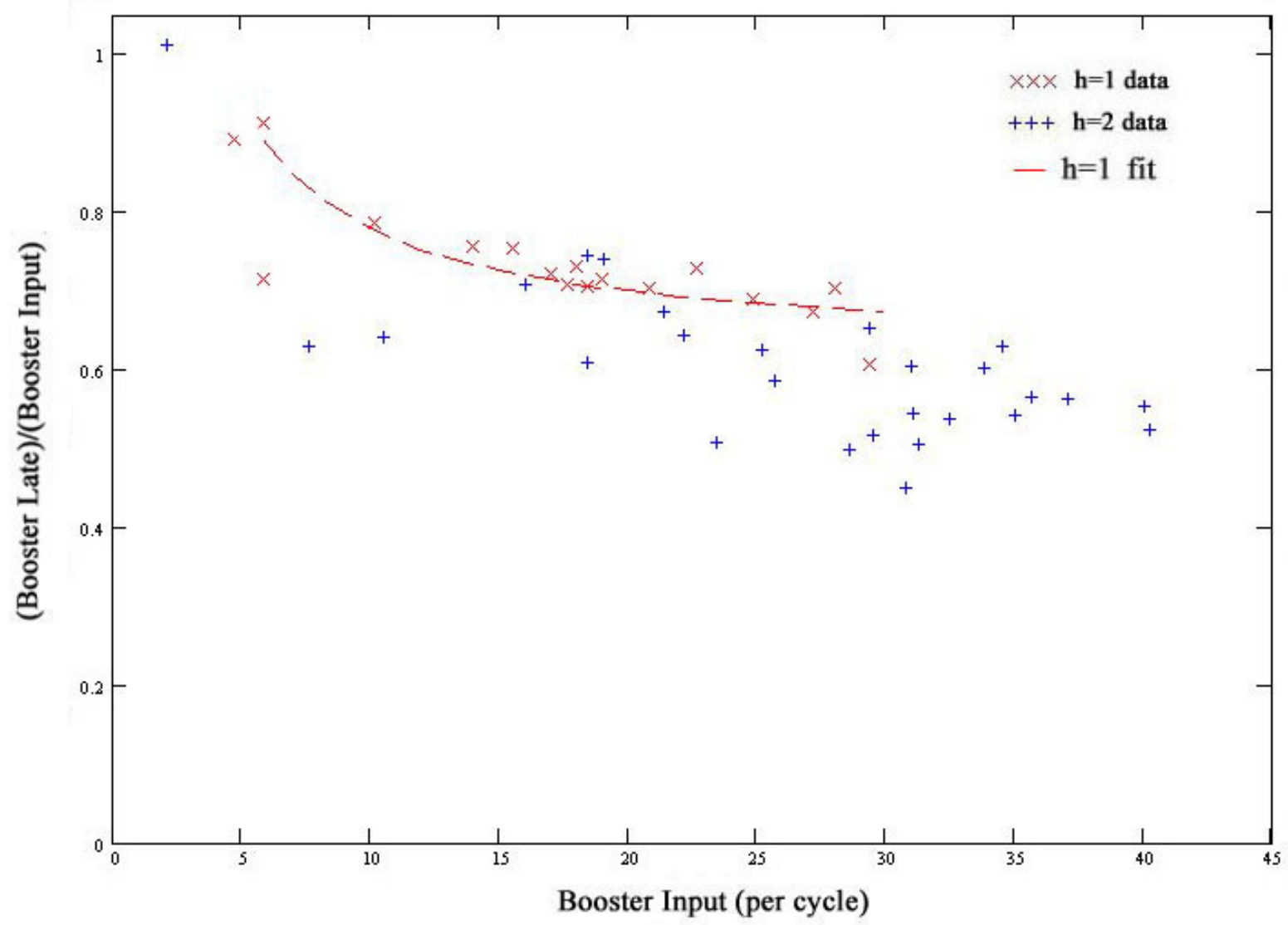

Figure 4: Booster Input (per cycle) vs. the Booster Efficiency (Late/Input) for h=1 (red) and h=2 (blue). The red line is the transformation of the linear fit for $\mathrm{h}=1$ in figure 3 into an efficiency function. The $\mathrm{h}=1$ points are taken from the $\mathrm{FY}{ }^{\prime} 98$, ' 01 , and ' 02 runs. The $\mathrm{h}=2$ points are taken from the FY' 95 , '96, and '97 runs.

A couple of unresolved issues that complicate the situation are:

1. There were no ALARA alarm constraints during the FY '98 $\mathrm{h}=1$ run. Why didn't the Booster intensity go any higher than it did during that run if there was no 'saturation', or flattening out, behavior in the Booster?

2. The Booster scalers during much of the ' 02 run were not calibrated correctly. Both the early and late numbers were reading 14\% low. In the data used in this note the scaler numbers have been adjusted to read 'correctly'. So, for most of the '98 run the injection loss appeared larger than it was and the injection ALARA limit was more of a constraint than it would appear to be from the figure 1. In practice, for most of the run, the injection ALARA limit was the major constraint. On the other hand, the transfer losses appeared too low, and so the transfer ALARA limit was artificially high (by 14\%) and not as much of a problem as it should have been. The acceleration losses also appeared lower by $14 \%$.

Figure 5 shows the per cycle late intensity versus the AGS CBM intensity divided by the number of transfers. The $\mathrm{h}=1$ data shows a pretty clear tendency towards saturation at late intensities higher than about $14 \mathrm{Tp}$ per cycle, whereas the $\mathrm{h}=2$ data looks linear, but does have a positive $y$-intercept. Additional $h=1$ data points were taken from the JEFF reports during the 2002 run in order to show the dependence more clearly in the region above 14 Tp. 


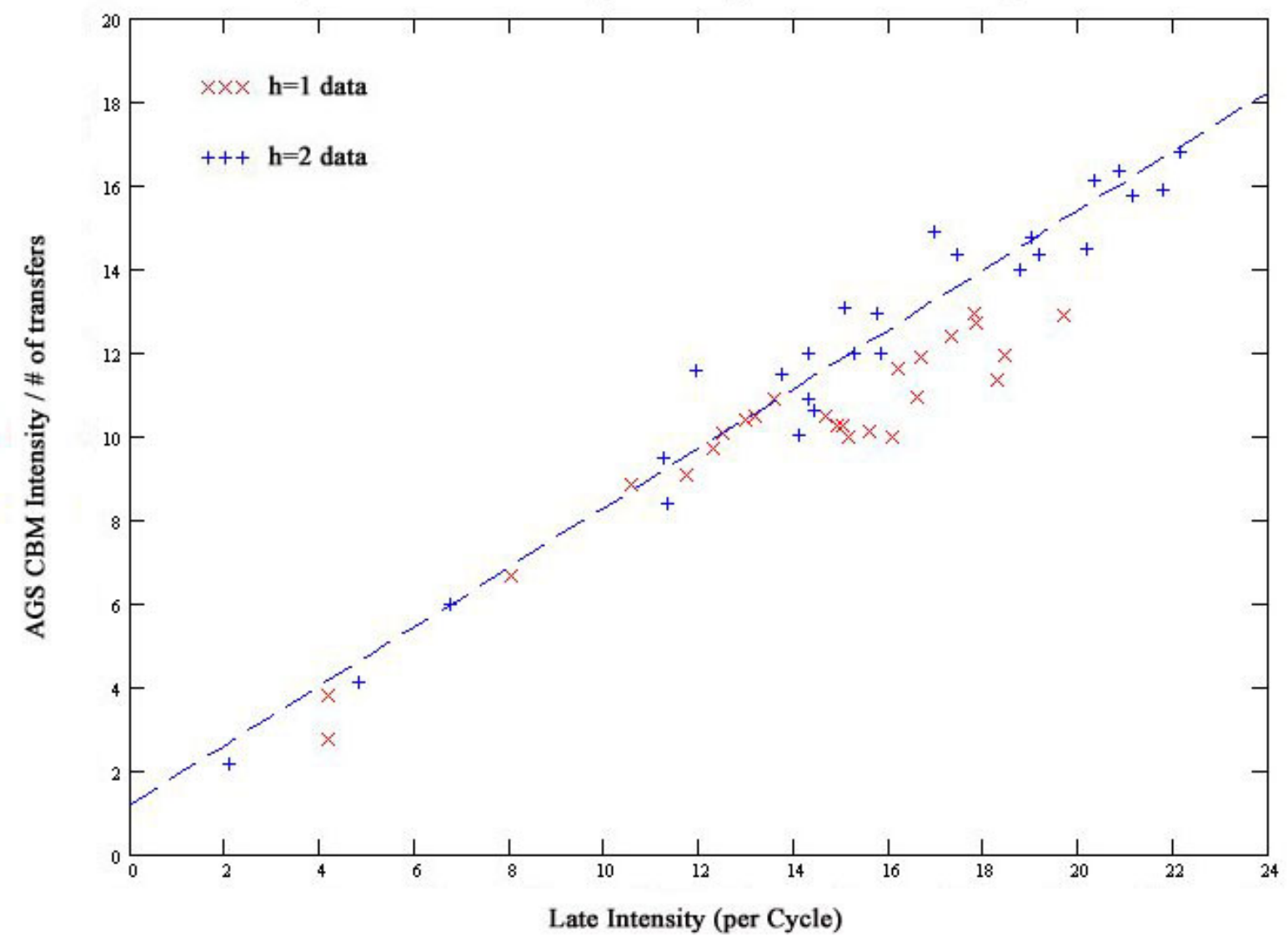

Figure 5: Booster Late per cycle vs. AGS CBM intensity divided by the number of transfers for $\mathrm{h}=1$ (red, 6 transfers) and $\mathrm{h}=2$ (blue, 4 transfers). The $\mathrm{h}=1$ points are taken from the FY ' 98 , '01, and '02 runs. The $\mathrm{h}=2$ points are taken from the FY' 95 , '96, and '97 runs. The blue dotted line is a linear fit to the $h=2$ data. Additional FY '02 points taken from JEFF reports.

Figure 6 shows a similar graph, but this time the total AGS CBM intensity is plotted against the total Booster late intensity. The linear fit for the $\mathrm{h}=1$ data for late intensities below $85 \mathrm{Tp}$ is quite good and passes the y-axis near zero. This implies that the efficiency has little intensity dependence over that range. The $\mathrm{h}=2$ case is quite linear but has a $\mathrm{y}$-intercept further from zero perhaps indicating more intensity dependence.

Figure 7 shows the efficiency plots for $\mathrm{h}=1$ and $\mathrm{h}=2$ with efficiency fits derived using a method similar to that used in figure 4 . The $\mathrm{h}=1$ efficiency gradually decreases to about $80 \%$ when Booster Late reaches 85-90 Tp, then drops sharply. The h=2 efficiency seems to drop gradually from around $90 \%$ at low intensity to $75-80 \%$ at high intensity.

The sharp drop in the efficiency at a Booster late intensity of 85-90 Tp is rather mysterious. Since there is no $h=2$ data for those intensities, there is nothing to suggest that this apparent saturation is specific to the $h=1$ setup. Such a severe saturation is not apparent in the Booster's performance, and is likely to be the strongest physical limiting factor to increasing the AGS intensity. As can be seen in figures 6 and 7, this strong saturation brings the losses to the FY'02 transfer ALARA limit in the region of the peak AGS CBM intensity. 


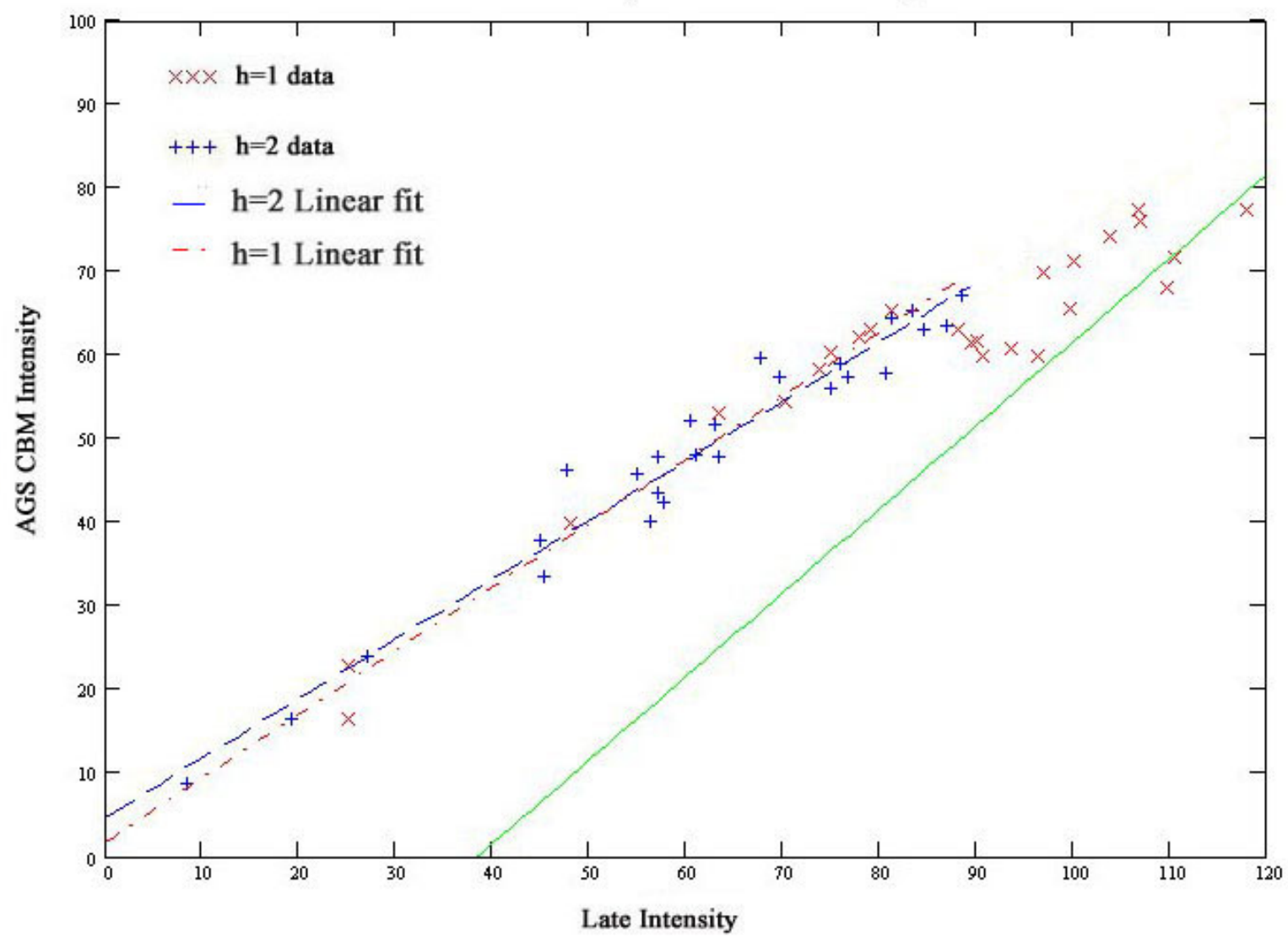

Figure 6: Booster Late vs. AGS CBM intensity for $\mathrm{h}=1$ (red, 6 transfers) and h=2 (blue, 4 transfers). The $\mathrm{h}=1$ points are taken from the $\mathrm{FY}$ ' 98 , ' 01 , and ' 02 runs. The $\mathrm{h}=2$ points are taken from the FY '95, '96, and '97 runs. The blue and red dotted lines are linear fits to the $h=2$ and $h=1$ data. The $\mathrm{h}=1$ fit was only done for late intensities below $85 \mathrm{Tp}$. Additional FY' 02 points taken from JEFF reports. The green line is the transfer ALARA limit for the 2002 run.

\section{Conclusion}

Although ALARA limits, even with a long repetition period, prohibit exploring the region beyond about 19-20 Tp per cycle with $\mathrm{h}=1$, they do not appear solely responsible for limiting the AGS intensity. There appears to be an intensity-related saturation effect associated with the transfer that is also limiting it. In the region where data exists for both the $h=1$ and $h=2$ setups as a function of total Booster late intensity vs. AGS CBM their efficiencies are roughly similar. So, there is little to suggest that the same thing wouldn't happen with the $h=2$ setup at these AGS CBM values. If fact, the transfer efficiency of the $\mathrm{h}=1$ setup may have less intensity dependence where data exist for both setups. This saturation effect may be the reason why the Booster Input did not regularly run above about $30 \mathrm{Tp}$ per cycle with $\mathrm{h}=1$ before the advent of ALARA alarms.

Although the Booster's performance is intensity dependent, there is no strong saturation obvious in the data like there is associated with the transfer. Since there is plenty of beam current available from the Linac, this suggests that Booster late could be raised beyond 19-20 Tp per cycle if the AGS could accept the additional beam. 
Booster Late Vs. Transfer Efficiency

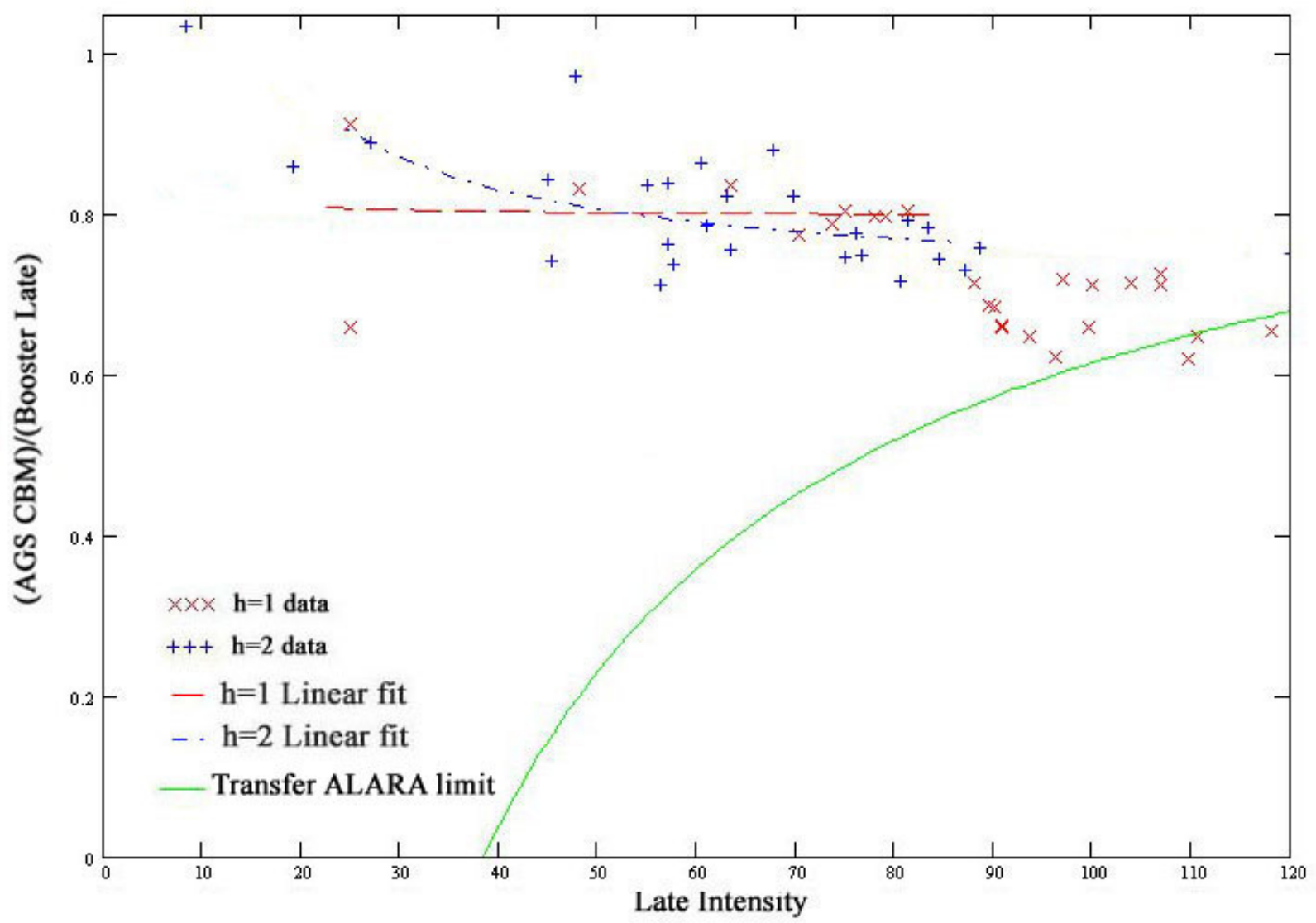

Figure 7: Booster Late vs. Transfer efficiency for $h=1$ (red, 6 transfers) and $h=2$ (blue, 4 transfers). The red (blue) line is the transformation of the linear fit for $\mathrm{h}=1(\mathrm{~h}=2)$ in figure 6 into an efficiency function. The efficiency function for $\mathrm{h}=1$ only looks at Booster Late below $85 \mathrm{Tp}$. The $\mathrm{h}=1$ points are taken from the FY '98, '01, and ' 02 runs. The $\mathrm{h}=2$ points are taken from the FY '95, '96, and '97 runs. Additional $\mathrm{FY}{ }^{\prime} 02 \mathrm{~h}=1$ points are taken from JEFF reports. The green line is the transfer ALARA limit for the 2002 run.

It should be noted that not much time is spent optimizing the machines at lower intensities. As a result, the data may be skewed somewhat in that a higher efficiency than indicated by the data may be possible at abnormally low intensities. It is also harder to measure the efficiency at lower intensities due to inaccuracies in the scaler calibrations and the small number of data points there.

\section{$\underline{\text { References: }}$}

HEP Setup Books for FY '95:

Book I: pg. 132, 133, 135

Book II: pg. 12, 72, 105, 116

Book III: pg. 120, 121

Book IV: pg. 17, 19, 20, 23, 33, 46

HEP Setup Books for FY'96

Book II: pg. 23, 69, 85, 113, 122, 139

Book III: pg. 17

HEP Setup Books for FY'98

Book I: pg. 67

Book II: pg. 93, 118 
HEP Setup Book FY ’99, pg. 119

HEP Setup Books for FY '00

Book I: pg. 132, 138, 150

Book II: pg. 2,8,17,18

HEP Setup Books for FY'02:

Book II: pg. 30, 38, 45, 115, 139

JEFF reports from the following dates in 2002: $\quad$ Mar 13, 16, 18, 20, and 21

Apr 30

May 15, 31

Jun 3, 7, and 9 